\title{
miR-132 serves as a diagnostic biomarker in gestational diabetes mellitus and its regulatory effect on trophoblast cell viability
}

\author{
Xuegui Zhou ${ }^{1 *}$, Cuiping Xiang ${ }^{1}$ and Xiaoxia Zheng $^{2}$
}

\begin{abstract}
Background: Gestational diabetes mellitus (GDM) leads to poor pregnancy outcomes. Strategies that improve trophoblast cell function are important methods for GDM treatment. This study aimed to investigate the expression and diagnostic potential of microRNA-132 (miR-132) in GDM patients, and further analyzed the effects of miR-132 on HTR-8/SVneo cell proliferation.

Methods: Quantitative real-time PCR was applied to estimate the expression of miR-132. A receiver operating characteristics curve (ROC) analysis was performed to evaluate the diagnostic value of serum miR-132 in GDM patients. In vitro regulation of miR-132 in trophoblast cell HTR-8/SVneo was achieved by cell transfection, and the effects of miR-132 on cell proliferation were assessed using CCK-8 assay.

Results: Expression of miR-132 was decreased in serum and placenta tissues in GDM patients compared with the healthy women. A negative correlation was found between the serum miR-132 levels and fasting blood glucose of the GDM patients. A ROC curve shown the serum miR-132 had considerable diagnostic accuracy with an area under the curve (AUC) of 0.898. High glucose (HG) treatment induced an inhibition in HTR-8/SVneo cell proliferation and the expression of miR-132. The overexpression of miR-132 in HTR-8/SVneo cells could markedly rescued the HG - induced suppressed cell proliferation.

Conclusion: All the data of this study revealed the reduced expression of miR-132 in serum and placenta tissues of GDM, and serum miR-132 serves a candidate biomarker in the diagnosis of GDM. miR-132 may act a protective role against GDM via enhancing the trophoblast cell proliferation.
\end{abstract}

Keywords: Gestational diabetes mellitus, MicroRNA-132, Trophoblast cell, Proliferation, Diagnosis

\section{Introduction}

Gestational diabetes mellitus (GDM) is a special kind of diabetes mellitus, which first occurs in pregnancy with impaired glucose tolerance [1]. Statistical data reveal that there is approximately $18.9 \%$ of pregnant women diagnosed with GDM in China [2]. GDM induces numerous complications and leads to poor pregnancy outcomes, including abortion, fetal malformation, fetal growth restriction, premature rupture of membrane and macrosomia

\footnotetext{
* Correspondence: xueguizhou2015c19@163.com

1 Department of Obstetrics, Binzhou People's Hospital, No. 515, Huanghe 7

Road, Binzhou, Shandong 256610, People's Republic of China

Full list of author information is available at the end of the article
}

[3]. Similar to general diabetes mellitus, hyperglycemia and insulin resistance are also the characteristics of GDM patients [4]. Thus, GDM has great potential to progress to type 2 diabetes mellitus (T2DM) if no any lifestyle, dietary or pharmacological intervention are administrated [5]. Thus, early diagnosis is very important for the women with GDM.

High glucose (HG) as one of the major characteristics of GDM has been reported to impair the normal development of placenta, resulting in the increases in abortion and fetus malformation [6]. Placenta is a pivotal organ for nutrition exchange between fetus and mother, its regular growth and development are premises and guarantees of a

(c) The Author(s). 2019 Open Access This article is distributed under the terms of the Creative Commons Attribution 4.0 International License (http://creativecommons.org/licenses/by/4.0/), which permits unrestricted use, distribution, and 
healthy pregnancy [7]. During the development placenta, normal function of trophoblast cells is one of the most critical events. The impairment in trophoblast cell proliferation can lead to the maldevelopment of placenta tissues $[8,9]$. However, the understanding about the mechanisms underlying the regulation of trophoblast cell biological function in GDM remains limited and urgently needs to be uncovered.

MicroRNAs (miRNAs) are a class of small noncoding RNAs and involved in numerous disease progressions, including the development of GDM [10, 11]. The dysregulation of miRNA has dramatic clinical significance in diagnosis and prognosis of human diseases, and can be used as efficient therapeutic targets due to their critical regulatory roles in various cellular processes, such as proliferation, migration, invasion and apoptosis [12-15]. MicroRNA-132 (miR-132) has been previously reported to be downregulated in GDM patients [16], but its clinical and biological function in GDM are rarely reported.

This study firstly investigated the expression of miR132 in serum and placenta tissues of GDM, and further evaluated its diagnostic potential for GDM patients. By using trophoblast cell HTR-8/SVneo, this study sought to uncover the functional role of miR-132 in the regulation of cell proliferation.

\section{Materials and methods}

\section{Patients and sample collection}

One hundred and eight GDM patients and 50 healthy pregnant women as a control group were enrolled in this study from Binzhou People's Hospital between 2013 and 2017. The diagnosis of GDM was determined following the guidelines of American Diabetes Association [17]. The cases with pre-gestational diabetes, multiple gestation accompanied with further complications and had medication were excluded from this study. Blood samples were collected from the participants at the 24-28 pregnancy weeks after an overnight fast, and fasting blood glucose was measured and recorded. Placental tissues were collected from the participants at the time of delivery, then stored in liquid nitrogen for further examinations. All the participants signed the informed consent for sample acquisition for research purposes. The protocols of this study were approved by the Ethics Committee of Binzhou People's Hospital.

\section{Cell culture and treatment}

Human trophoblast cell lines HRT-8/SVneo and BeWo was obtained from the Chinese Academy of Sciences Cell Bank (Shanghai, China). HRT-8/SVneo was cultured in Dulbecco's modified Eagle's medium (DMEM; Invitrogen, MA, USA) supplemented with $1000 \mathrm{mg} / \mathrm{L}$ glucose and 10\% fetal bovine serum (FBS; Gibco, CA, USA), and BeWo was cultured in cultured in F-12 medium
(Gibco, CA, USA) added with $10 \% \mathrm{FBS}$ at $37^{\circ} \mathrm{C}$ in a humidified atmosphere containing $5 \% \mathrm{CO}_{2}$. The cells were divided into two groups, including HG treated group and normal cell group. Cells in the HG group were cultured in a HG medium with the glucose concentration of $25 \mathrm{mM}$, while the cells in the normal group were incubated in the normal culture medium (glucose concentration of $5 \mathrm{mM})$.

\section{Cell transfection}

Cell transfection vectors, including miR-132 mimic, miR-132 inhibitor and the separate negative control (mimic NC and inhibitor NC), were purchased from GenePharma (Shanghai, China). These vectors were respectively transfected into the trophoblast cells using Lipofectamine 3000 (Invitrogen, Carlsbad, CA, USA) following the manufactures' instructions. After $48 \mathrm{~h}$ of the transfection, the cells were used for the next experiments.

\section{RNA extraction and quantitative real-time PCR (qRT-PCR)}

Total RNA was extracted using the TRIzol reagent (Invitrogen, Carlsbad, CA, USA) and its quality was estimated using a NanoDrop 2000 (Thermo Fisher Scientific, Waltham, MA, USA). cDNA was synthesized from the RNA according to a PrimeScript RT reagent kit (TaKaRa, Shiga, Japan) as per the protocols of manufactures. The expression of miR-132 was examined using qRT-PCR, which was carried out using a 7300 Real-Time PCR System (Applied Biosystems, USA) and a SYBR green I Master Mix kit (Invitrogen, Carlsbad, CA, USA). The final expression values were calculated using the $2^{-\Delta \Delta C t}$ method and normalized to U6.

\section{Cell proliferation assay}

After cell transfection, the trophoblast cells were seeded into 96-well culture plates for the cell proliferation examination using a Cell Counting Kit-8 (CCK-8; Beyotime, Shanghai, China). The cells were cultured at $37^{\circ} \mathrm{C}$ for 3 days, and the CCK- 8 reagent was added into the wells every $24 \mathrm{~h}$ following with a $4 \mathrm{~h}$ further incubation. The cell proliferation was evaluated by reading the absorbance of cell cultures at $450 \mathrm{~nm}$ using a microplate reader (Molecular Devises, CA, USA).

\section{Statistical analysis}

Data in this study were presented as mean \pm SD and analyzed using SPSS 21.0 software (SPSS Inc., Chicago, IL) and GraphPad Prism 7.0 software (GraphPad Software, Inc., USA). Comparisons between groups were assessed using Student's t test or one-way ANOVA. Pearson correlation analysis was performed to estimate the correlations between parameters. A receiver operating characteristics curve (ROC) was plotted to evaluate the diagnostic value 
of miR-132 in the patients with GDM. A result with $P<$ 0.05 was considered statistically significant.

\section{Results}

\section{Baseline characteristics of the participants}

A total of 158 pregnant women were enrolled in our study, including 50 healthy controls and 108 GDM patients. The demographic and clinical features of the participants were compared and no significant difference was observed in age, BMI and pregnancy weeks between the two groups (all $P>0.05$, Table 1). Compared with the healthy women, the fasting blood glucose was markedly higher in the women with GDM $(P<0.001)$.

\section{Expression of miR-132 in serum and placenta tissues of GDM}

As shown in Fig. 1a, we observed a significant decrease in serum expression of miR-132 in the GDM patients compared with the healthy controls $(P<0.001)$. Placental tissue of the participants during delivery were collected to further examine the expression patterns of miR-132. Similar to the serum data, the expression of miR-132 in the placental tissues was also obviously decreased in the GDM patients in a comparison to the healthy controls $(P<0.001$, Fig. $1 b)$. Increased blood glucose is a characteristic of GDM, and the fasting blood glucose was as expected elevated in the GDM patients in this study. This study further found a negative correlation of the serum miR-132 expression with the patients' fasting blood glucose levels $(r=-0.490, P<0.001$, Fig. 1 c).

\section{Diagnostic value of miR-132 for patients with GDM}

Since the serum expression of miR-132 in GDM patients was remarkably different from the healthy controls, the diagnostic potential of miR-132 was further investigated. A ROC curve (Fig. 2) constructed based on serum miR132 levels revealed that the area under the curve (AUC) was 0.898 , indicating the relative high diagnostic accuracy of miR-132 for the differentiation between GDM patients and healthy women. The diagnostic sensitivity and specificity were respectively 84.3 and $88.0 \%$ at a cutoff value of $1.995 \times 10^{-4}$.
HG suppresses trophoblast cell viability and the expression of miR-132

HG in GDM patients leads to inhibited trophoblast cell proliferation, thus resulting in the impaired placental development. From Fig. 3a, the cell proliferation of trophoblast cells cultured in HG medium was as expected significantly suppressed compared with the normal controls $(P<0.05)$. Similar to the serum expression data in GDM patients, the expression of miR-132 was also reduced by HG in trophoblast cells when compared to the untreated cells $(P<0.001$, Fig. 3b).

\section{Overexpression of miR-132 contributes trophoblast cell viability upon $\mathrm{HG}$ treatment}

Since the expression of miR-132 was inhibited in trophoblast cells upon HG treatment, this study sought to investigate whether the regulation of miR-132 could lead to any change of cell viability of the trophoblast cells. By cell transfection, the expression of miR-132 was upregulated by the miR-132 mimic, but was downregulated by the miR-132 inhibitor in trophoblast cells (all $P<0.001$, Fig. 4a). According to CCK- 8 assay, we found that the overexpression of miR-132 could promote the trophoblast cell proliferation, while the knockdown of miR-132 resulted in the inhibited cell proliferation ability (all $P<0.05$, Fig. 4b).

\section{Discussion}

GDM is a healthy burden among pregnant women and can lead to poor pregnancy outcomes. HG as a characteristic of GDM, inducing the inhibited trophoblast cell proliferation and thereby resulting in the impaired development of placenta. This study investigated the expression of miR-132 in GDM patients and explored the regulatory effects of miR-132 on cell proliferation of trophoblast cell upon HG treatment. The data of this study shown that the expression of miR-132 in serum and placenta tissues was decreased in the GDM patients compared with the healthy pregnant women. The serum miR-132 expression was negatively correlated with the fasting blood glucose of the patients and had relative high diagnostic accuracy for the patients with GDM. HG induced the suppressed cell viability and reduced miR132 expression in both HTR-8/SVneo and BeWo cells. By in vitro regulation, the inhibited trophoblast cell

Table 1 Demographic and clinical characteristics of the women included in this study

\begin{tabular}{llll}
\hline Parameters & Healthy controls $(n=50)$ & GDM patients $(n=108)$ & $P$ value \\
\hline Age (years, mean $\pm \mathrm{SD})$ & $30.10 \pm 2.79$ & $30.89 \pm 3.45$ & 0.159 \\
BMI $\left(\mathrm{kg} / \mathrm{m}^{2}\right.$, mean $\left.\pm \mathrm{SD}\right)$ & $20.10 \pm 1.65$ & $22.82 \pm 2.57$ & 0.064 \\
Pregnancy weeks (weeks, mean $\pm \mathrm{SD})$ & $25.32 \pm 1.45$ & $25.20 \pm 1.23$ & 0.602 \\
Fasting blood glucose $(\mathrm{mM} / \mathrm{L}$, mean $\pm \mathrm{SD})$ & $4.43 \pm 0.27$ & $6.31 \pm 0.31$ & $<0.001$
\end{tabular}

GDM gestational diabetes mellitus; BMl, body mass index 


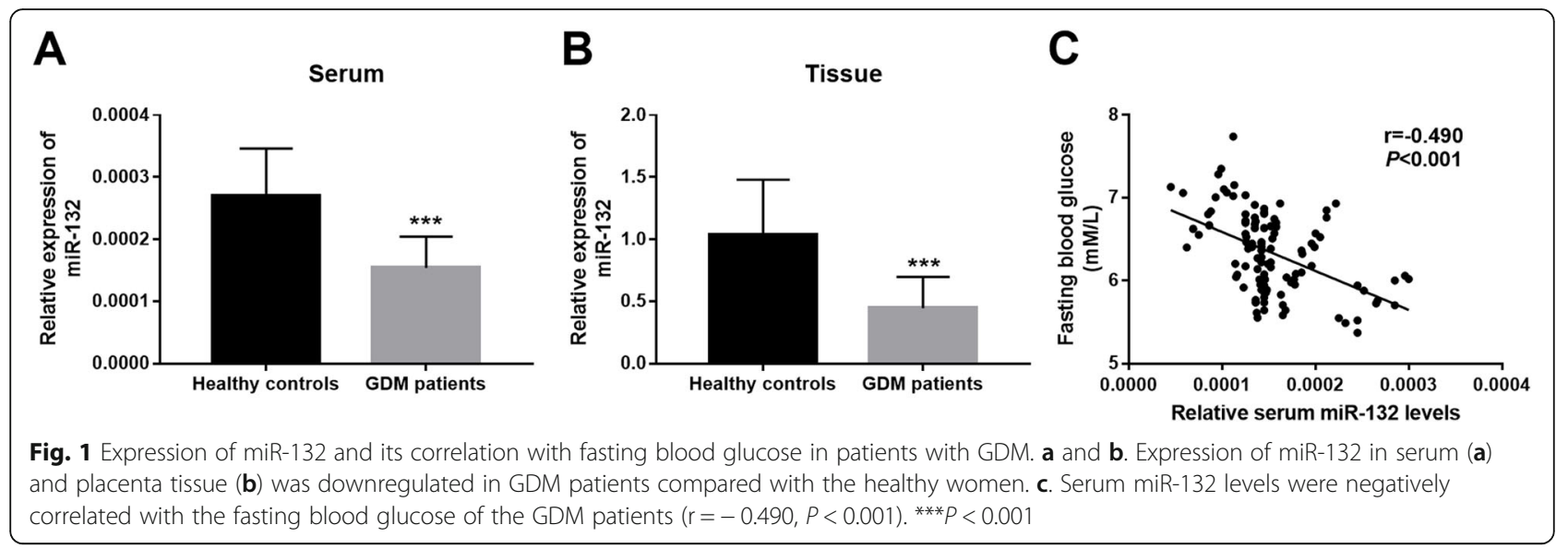

proliferation by HG was enhanced by the overexpression of miR-132.

Numerous miRNAs have been found to be abnormally expressed in various human diseases [18, 19]. These aberrant miRNAs are highlighted for their clinical applications and therapeutic potentials in the disease development and progression [20]. For example, serum expression of miR-30d has been reported to be downregulated in acute heart failure patients compared with the healthy controls, and the abnormal
miR-30d was determined as a candidate diagnostic and prognostic biomarker for this acute disease [21]. Increased expression of miR-483-5p was detected in esophageal squamous cell carcinoma tissues, and its dysregulation was demonstrated to serve as an independent prognostic biomarker of this malignancy [22]. In type 2 diabetes mellitus patients, serum elevated miR-7 was proved to be a promising diagnostic biomarker and its expression could be used as a potential predictor of microvascular complications [23]. For

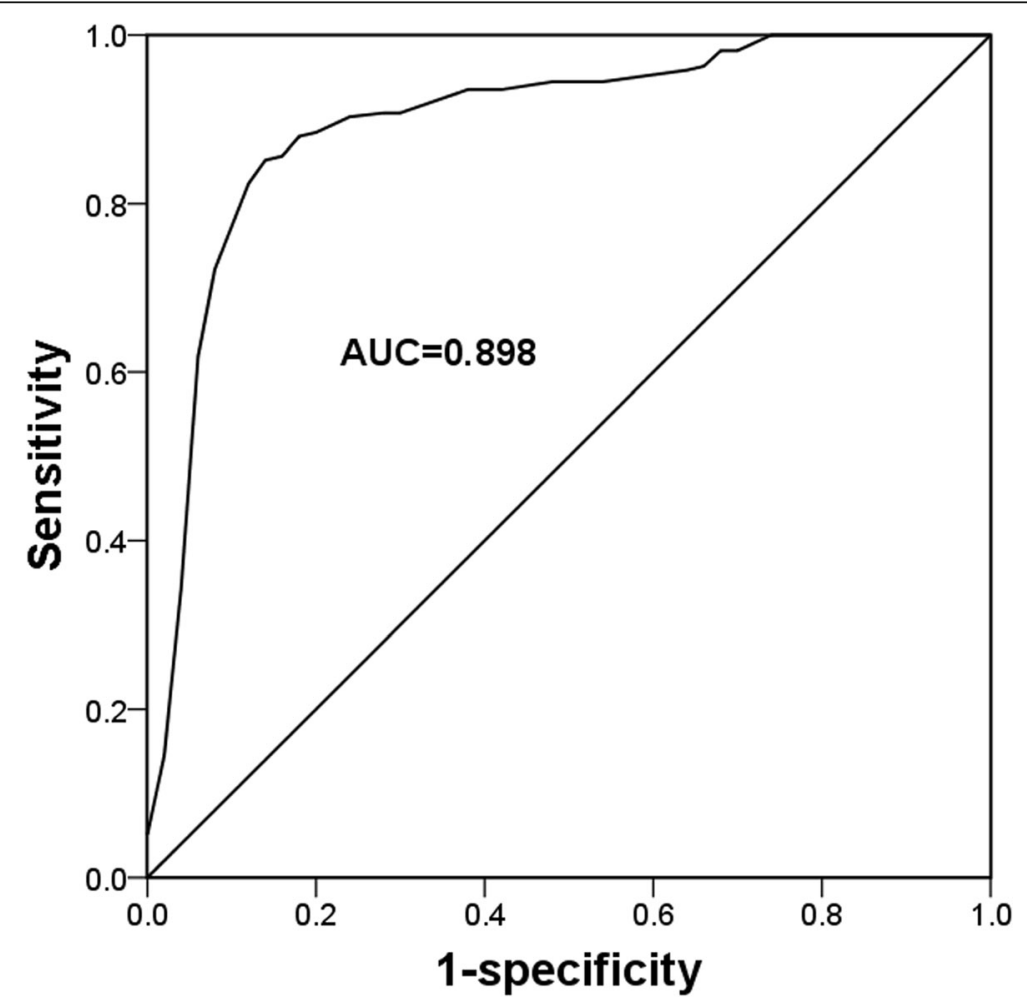

Fig. 2 A ROC curve based on serum miR-132 in GDM patients. The area under the curve (AUC) was 0.898 for serum miR-132 expression to distinguish GDM cases from healthy pregnant women 

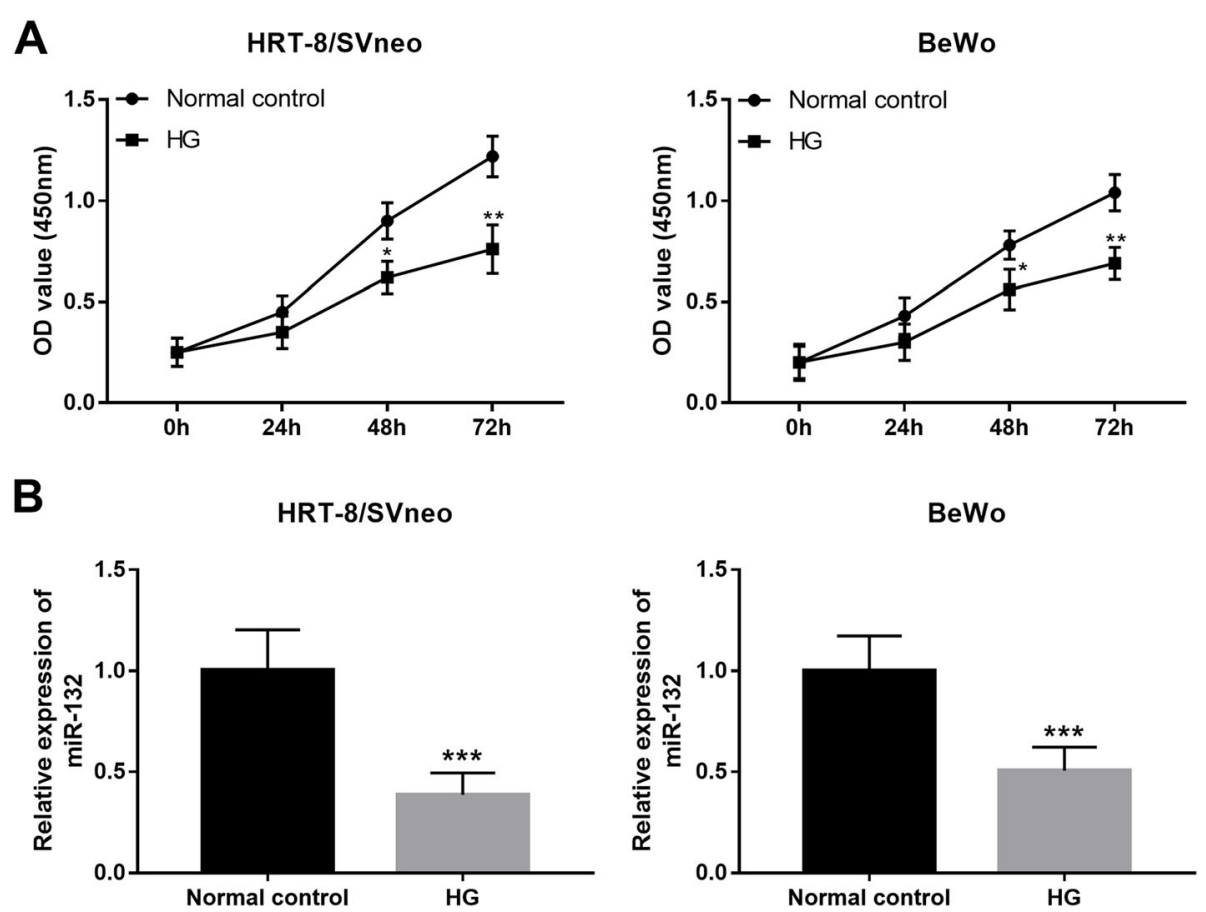

Fig. $3 \mathrm{HG}$ suppressed cell proliferation (a) and inhibited the expression of miR-132 (b) in two trophoblast cell lines HTR-8/SVneo and BeWo. HG, high glucose; ${ }^{*} P<0.05,{ }^{* *} P<0.01,{ }^{* *} P<0.001$

the patients with GDM, some miRNAs with aberrant expression profiles have also been reported, such as miR-20a-5p [24], miR-137 [25] and miR-98 [26]. In the present study, we found the significantly decreased expression of miR-132 in the serum and placenta tissues in GDM patients compared with the healthy pregnant women. Moreover, a negative correlation was demonstrated between the serum miR-132 levels and the fasting blood glucose concentration in the patients with GDM. Thus, we believed that the aberrant miR-132 might also play an important role in the pathogenesis of GDM.

Serum examination for various biomarkers is an established well diagnostic strategy for human diseases, including GDM [27]. MiRNAs are stable in serum and have been described as a group of pivotal diagnostic candidates for numerous human diseases, such as malignancies [28], diabetes mellitus [29] and also GDM [24]. Given the decreased serum expression of miR-132 in GDM patients, a ROC curve based on miR-132 was plotted and revealed that the reduced miR-132 expression might be a potential diagnostic biomarker for GDM patients with relative high sensitivity and specificity. Serum aberrant expression of miR-132 has previously reported to serve as a candidate biomarker in some other diseases, including hepatocellular carcinoma [30] and mild cognitive impairment [31]. The miRNAs, including miR132, are released by cells into different body fluids especially the blood, and exist stably with high resistance to harsh conditions and RNase digestion [32]. Thus, we believed that serum miR-132 may serve as a useful biomarker for human GDM.

As we all known that the regular growth and development of placenta is one of the most important step in a healthy pregnancy. However, the occurrence of GDM always along with the maldevelopment of placenta [6]. HG, as a characteristic of GDM, leads to the impaired function of trophoblast cells, thereby suppressing the normal development of placenta [33]. In the current study, the proliferation of trophoblast cell HTR-8/SVneo and BeWo was obviously inhibited by the HG treatment, which is consistent with the previous reports [33]. Additionally, HG treatment was also found to result in an inhibition in the expression of miR-132 in the trophoblast cells. Furthermore, the gain-of-function analysis indicated that the suppressed trophoblast cell proliferation induced by HG could be rescued by the overexpression of miR-132. miR-132 has been previously reported to regulate cell proliferation in pancreatic cancer [34] and laryngeal squamous cell carcinoma [35]. Thus, we considered that miR-132 might exert a protective role against GDM by enhancing the trophoblast cell proliferation. Zhang et al. has reported that miR-132 could facilitate cell proliferation of pancreatic cancer cells by targeting phosphatase and tensin homolog (PTEN) [34]. PETN is a major upstream inhibitor of the PI3K/AKT 
A

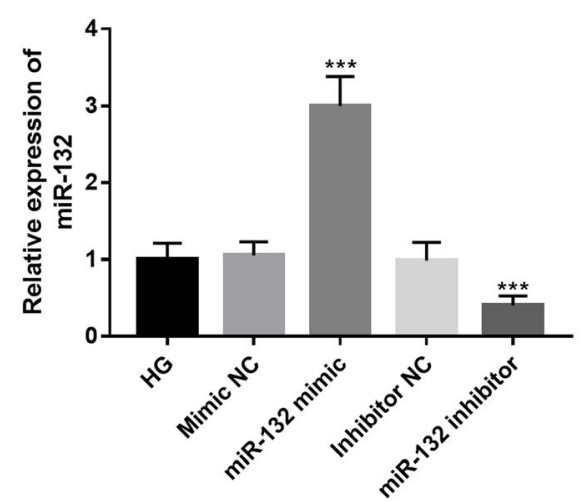

B

HRT-8/SVneo

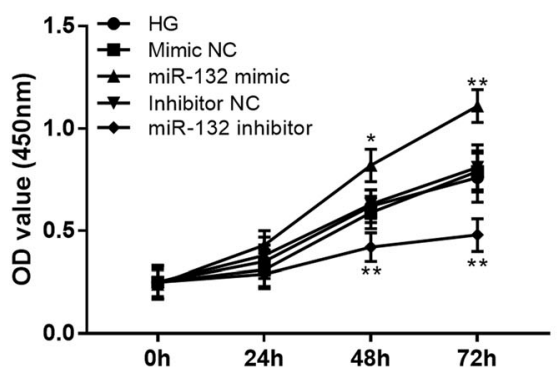

BeWo

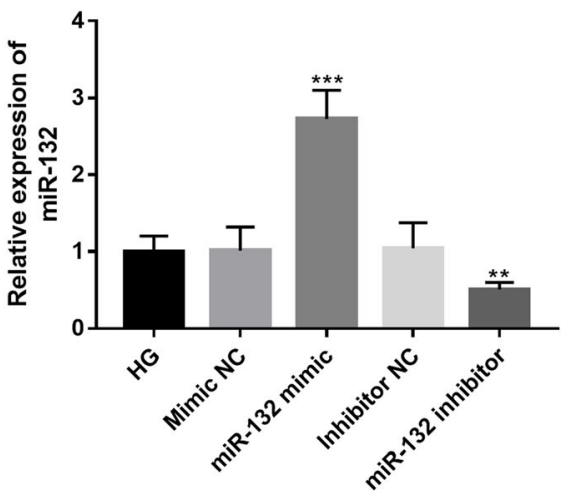

BeWo

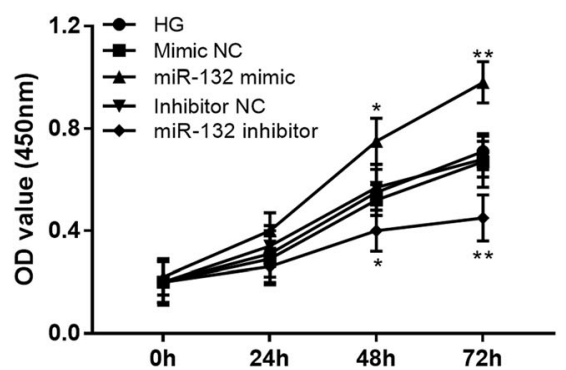

Fig. 4 Effects of miR-132 on cell proliferation of trophoblast cells upon HG treatment. a. Expression of miR-132 was upregulated by the miR-132 mimic, but was downregulated by the miR-132 inhibitor in both HTR-8/SVneo and BeWo cell lines. $\mathbf{b}$. The upregulation of miR-132 promoted cell proliferation, while the downregulation of miR-132 inhibited cell proliferation in HTR-8/SVneo and BeWo cells. HG, high glucose; ${ }^{*} P<0.05$, ${ }^{* *} P<0.01,{ }^{* * *} P<0.001$

signaling, and has been identified as a critical molecule in GDM [36]. Li et al. found that plasma PTEN levels was associated with insulin resistance of GDM [37]. In addition, the increased expression of PTEN mediates the regulatory effects of miR-21 [38] and miR-144 [39] on the biological function of trophoblastic cells. Thus, we suspected that protective action of miR-132 in GDM might also achieved through targeting PTEN. The precise mechanisms underlying the role of miR-132 in GDM need to be investigated in future studies.

\section{Conclusion}

Taken together, the data of this study demonstrated that the expression of miR-132 in serum and placenta tissues is reduced in the patients with GDM and serum miR132 expression serves a promising non-invasive diagnostic biomarker for the screening of GDM cases. In addition, the upregulation of miR-132 can abrogate the inhibiting effects of HG on trophoblast cell proliferation, indicating that miR-132 may play a protective role in GDM through promoting trophoblast cell viability.

\section{Abbreviations}

AUC: Area under the curve; CCK-8: Cell counting kit-8; DMEM: Dulbecco's modified Eagle's medium; FBS: Fetal bovine serum; GDM: Gestational diabetes mellitus; HG: High glucose; miR-132: microRNA-132; miRNAs: microRNAs; qRT-PCR: quantitative real-time PCR; ROC: Receiver operating characteristics curve; T2DM: Type 2 diabetes mellitus

\section{Acknowledgements}

Not applicable.

\section{Authors' contributions}

XZ made substantial contributions to conception and design, analysis and interpretation of data, and preparation of the manuscript. CX and XZ have been involved in acquisition of data. All authors read and approved the final manuscript.

\section{Funding}

Not applicable.

\section{Availability of data and materials}

All data generated during this study are included in this published article.

\section{Ethics approval and consent to participate}

All the participants signed the informed consent for sample acquisition for research purposes. The protocols of this study were approved by the Ethics Committee of Binzhou People's Hospital.

\section{Consent for publication}

Not applicable.

\section{Competing interests}

The authors declare that they have no competing interests. 


\section{Author details}

'Department of Obstetrics, Binzhou People's Hospital, No. 515, Huanghe 7 Road, Binzhou, Shandong 256610, People's Republic of China. ${ }^{2}$ Department of Obstetrics, Binzhou Center Hospital, Binzhou, Shandong 251700, People's Republic of China.

Received: 29 July 2019 Accepted: 9 October 2019

Published online: 25 October 2019

\section{References}

1. Spaight C, Gross J, Horsch A, Puder JJ. Gestational diabetes mellitus. Endocr Dev. 2016;31:163-78.

2. Wei $Y$, Yang $H$, Zhu W, Yang H, Li H, Yan J, et al. International Association of Diabetes and Pregnancy Study Group criteria is suitable for gestational diabetes mellitus diagnosis: further evidence from China. Chin Med J. 2014; 127(20):3553-6.

3. Miao M, Dai M, Zhang Y, Sun F, Guo X, Sun G. Influence of maternal overweight, obesity and gestational weight gain on the perinatal outcomes in women with gestational diabetes mellitus. Sci Rep. 2017;7(1):305.

4. Law KP, Zhang $H$. The pathogenesis and pathophysiology of gestational diabetes mellitus: Deductions from a three-part longitudinal metabolomics study in China. Clin Chim Acta. 2017:468:60-70.

5. Tanase-Nakao K, Arata N, Kawasaki M, Yasuhi I, Sone H, Mori R, et al. Potential protective effect of lactation against incidence of type 2 diabetes mellitus in women with previous gestational diabetes mellitus: A systematic review and meta-analysis. Diabetes Metab Res Rev. 2017:33(4):e2875.

6. Edu A, Teodorescu C, Dobjanschi CG, Socol ZZ, Teodorescu V, Matei A, et al. Placenta changes in pregnancy with gestational diabetes. Rom J Morphol Embryol. 2016;57(2):507-12.

7. Kaiser J. Reproductive biology. Gearing up for a closer look at the human placenta. Science. 2014;344(6188):1073.

8. Zong S, Li C, Luo C, Zhao X, Liu C, Wang K, et al. Dysregulated expression of IDO may cause unexplained recurrent spontaneous abortion through suppression of trophoblast cell proliferation and migration. Sci Rep. 2016:6:19916.

9. Tian FJ, Qin CM, Li XC, Wu F, Liu XR, Xu WM, et al. Decreased stathmin-1 expression inhibits trophoblast proliferation and invasion and is associated with recurrent miscarriage. Am J Pathol. 2015;185(10):2709-21.

10. Muralimanoharan S, Maloyan A, Myatt L. Mitochondrial function and glucose metabolism in the placenta with gestational diabetes mellitus: role of miR-143. Clin Sci. 2016;130(11):931-41.

11. Li J, Liang $Y$, Lv H, Meng H, Xiong G, Guan X, et al. miR-26a and miR-26b inhibit esophageal squamous cancer cell proliferation through suppression of c-MYC pathway. Gene. 2017;625:1-9.

12. Qiu Z, Li H, Wang J, Sun C. miR-146a and miR-146b in the diagnosis and prognosis of papillary thyroid carcinoma. Oncol Rep. 2017;38(5):2735-40.

13. Liu W, Zhao X, Zhang YJ, Fang GW, Xue Y. MicroRNA-375 as a potential serum biomarker for the diagnosis, prognosis, and chemosensitivity prediction of osteosarcoma. J Int Med Res. 2018;46(3):975-83.

14. He W, Huang L, Li M, Yang Y, Chen Z, Shen X. MiR-148b, MiR-152/ALCAM Axis regulates the proliferation and invasion of pituitary adenomas cells. Cell Physiol Biochem. 2017;44(2):792-803.

15. Wang HF, Wang WH, Zhuang HW, Xu M. MiR-429 regulates the proliferation and apoptosis of nephroblastoma cells through targeting c-myc. Eur Rev Med Pharmacol Sci. 2018;22(16):5172-9.

16. Zhao C, Dong J, Jiang T, Shi Z, Yu B, Zhu Y, et al. Early second-trimester serum miRNA profiling predicts gestational diabetes mellitus. PLoS One. 2011;6(8):e23925

17. American DA. Diagnosis and classification of diabetes mellitus. Diabetes Care. 2012;35(Suppl 1):S64-71.

18. Pishavar E, Behravan J. miR-126 as a therapeutic agent for diabetes mellitus. Curr Pharm Des. 2017;23(22):3309-14.

19. Vishnoi A, Rani S. MiRNA biogenesis and regulation of diseases: an overview. Methods Mol Biol. 2017;1509:1-10.

20. Kumar S, Vijayan M, Bhatti JS, Reddy PH. MicroRNAs as peripheral biomarkers in aging and age-related diseases. Prog Mol Biol Transl Sci. 2017; 146:47-94.

21. Xiao J, Gao R, Bei Y, Zhou Q, Zhou Y, Zhang H, et al. Circulating miR-30d predicts survival in patients with acute heart failure. Cell Physiol Biochem. 2017;41(3):865-74
22. Xue L, Nan J, Dong L, Zhang C, Li H, Na R, et al. Upregulated miR-483-5p expression as a prognostic biomarker for esophageal squamous cell carcinoma. Cancer Biomarkers. 2017;19(2):193-7.

23. Wan S, Wang J, Wang J, Wu J, Song J, Zhang CY, et al. Increased serum miR-7 is a promising biomarker for type 2 diabetes mellitus and its microvascular complications. Diabetes Res Clin Pract. 2017;130:171-9.

24. Pheiffer $C$, Dias S, Rheeder P, Adam S. Decreased expression of circulating miR-20a-5p in south African women with gestational diabetes mellitus. Mol Diagn Ther. 2018;22(3):345-52.

25. Peng HY, Li HP, Li MQ. High glucose induces dysfunction of human umbilical vein endothelial cells by upregulating miR-137 in gestationa diabetes mellitus. Microvasc Res. 2018;118:90-100.

26. Cao JL, Zhang L, Li J, Tian S, Lv XD, Wang XQ, et al. Up-regulation of miR-98 and unraveling regulatory mechanisms in gestational diabetes mellitus. Sci Rep. 2016:6:32268.

27. Chen $T$, He P, Tan Y, Xu D. Biomarker identification and pathway analysis of preeclampsia based on serum metabolomics. Biochem Biophys Res Commun. 2017:485(1):119-25.

28. Wang SY, Li Y, Jiang YS, Li RZ. Investigation of serum miR-411 as a diagnosis and prognosis biomarker for non-small cell lung cancer. Eur Rev Med Pharmacol Sci. 2017;21(18):4092-7.

29. Yang Z, Chen H, Si H, Li X, Ding X, Sheng Q, et al. Serum miR-23a, a potential biomarker for diagnosis of pre-diabetes and type 2 diabetes. Acta Diabetol. 2014:51(5):823-31.

30. Wang F, Wang J, Ju L, Chen L, Cai W, Yang J. Diagnostic and prognostic potential of serum miR-132/212 cluster in patients with hepatocellular carcinoma. Ann Clin Biochem. 2018;55(5):576-82.

31. Xie B, Zhou H, Zhang R, Song M, Yu L, Wang L, et al. Serum miR-206 and miR-132 as potential circulating biomarkers for mild cognitive impairment. J Alzheimers Dis. 2015;45(3):721-31.

32. Chen $X$, Ba $Y$, Ma L, Cai $X$, Yin $Y$, Wang $K$, et al. Characterization of microRNAs in serum: a novel class of biomarkers for diagnosis of cancer and other diseases. Cell Res. 2008:18(10):997-1006.

33. Peng HY, Li MQ, Li HP. High glucose suppresses the viability and proliferation of HTR8/SVneo cells through regulation of the miR137/ PRKAA1/IL6 axis. Int J Mol Med. 2018;42(2):799-810.

34. Zhang $H$, Liu A, Feng $X$, Tian L, Bo W, Wang $H$, et al. MiR-132 promotes the proliferation, invasion and migration of human pancreatic carcinoma by inhibition of the tumor suppressor gene PTEN. Prog Biophys Mol Biol. 2017: S0079610717302031.

35. Lian R, Lu B, Jiao L, Li S, Wang H, Miao W, et al. MiR-132 plays an oncogenic role in laryngeal squamous cell carcinoma by targeting FOXO1 and activating the PI3K/AKT pathway. Eur J Pharmacol. 2016;792:1-6.

36. He Y, Bai J, Liu P, Dong J, Tang Y, Zhou J, et al. miR-494 protects pancreatic beta-cell function by targeting PTEN in gestational diabetes mellitus. EXCLI 2017:16:1297-307.

37. Li YY, Xiao R, Li CP, Huangfu J, Mao JF. Increased plasma levels of FABP4 and PTEN is associated with more severe insulin resistance in women with gestational diabetes mellitus. Med Sci Monit. 2015;21:426-31.

38. Lou CX, Zhou XT, Tian QC, Xie HQ, Zhang JY. Low expression of microRNA21 inhibits trophoblast cell infiltration through targeting PTEN. Eur Rev Med Pharmacol Sci. 2018;22(19):6181-9.

39. Xiao J, Tao T, Yin Y, Zhao L, Yang L, Hu L. miR-144 may regulate the proliferation, migration and invasion of trophoblastic cells through targeting PTEN in preeclampsia. Biomed Pharmacother. 2017:94:341-53.

\section{Publisher's Note}

Springer Nature remains neutral with regard to jurisdictional claims in published maps and institutional affiliations. 